\title{
Computed tomography optimised fluoroscopy guidance for transcatheter mitral therapies
}

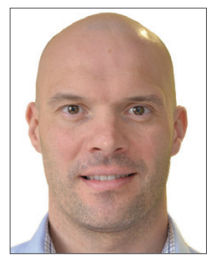

Nicolas M. Van Mieghem ${ }^{1 *}$, MD, PhD; Ramón Rodríguez-Olivares ${ }^{1}$, MD; Ben C. Ren ${ }^{1}, \mathrm{MD}, \mathrm{PhD}$; Lennart van Gils ${ }^{1}, \mathrm{MD}$; Annemarie Maugenest ${ }^{1}, \mathrm{MSc}$; Marcel L. Geleijnse' ${ }^{1}, \mathrm{MD}, \mathrm{PhD}$; Ricardo P.J. Budde ${ }^{2}, \mathrm{MD}, \mathrm{PhD}$; Johan Vogelaar ${ }^{3}, \mathrm{BSc}$; Luc Verstraeten $^{3}$, BSc; Peter P. de Jaegere ${ }^{1}, \mathrm{MD}, \mathrm{PhD}$

1. Department of Cardiology, Thoraxcenter, Erasmus Medical Center, Rotterdam, The Netherlands; 2. Department of Radiology, Thoraxcenter, Erasmus Medical Center, Rotterdam, The Netherlands; 3. 3mensio Medical Imaging BV, Maastricht, The Netherlands

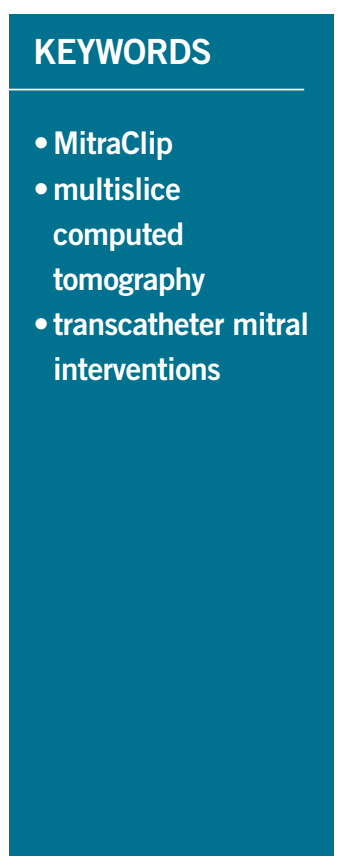

\begin{abstract}
Aims: Our aim was to illustrate the pragmatic use of pre-procedural multislice computed tomography (MSCT) to facilitate fluoroscopy guidance of transcatheter mitral valve interventions.

Methods and results: A dedicated software package (3mensio Structural Heart) is used to analyse MSCT studies and localise anatomical entities by fluoroscopy which would otherwise be invisible (e.g., interatrial septum, paravalvular leaks, mitral leaflets), and to provide optimal C-arm gantry angles to facilitate crucial steps of catheter-based mitral interventions. For any given anatomical structure that has been identified by MSCT scan, a line of perpendicularity can be drawn representing an infinite combination of RAO-LAO with cranial-caudal angles. Safety and ergonomic considerations drive the selected angulation to be used in the cathlab. The location of the fossa ovalis can be projected onto the fluoroscopy screen to help direct the needle for transseptal puncture. For MitraClip implantations a C-arm gantry projection that is either coaxial or perpendicular to the mitral coaptation plane helps to orientate the clip before entering the left ventricle to grasp the mitral leaflets. A periprosthetic mitral leak can be localised relative to the prosthesis in the proposed C-arm angle. Pre-procedural MSCT is thus complementary to transoesophageal echocardiography for transcatheter mitral interventions.
\end{abstract}

Conclusions: Determination of optimal C-arm angulations helps localise anatomical entities by fluoroscopy and may expedite complex mitral interventions.

\footnotetext{
*Corresponding author: Department of Interventional Cardiology, Thoraxcenter, Erasmus MC, Room Bd 171, 's Gravendijkwal 230,3015 CE Rotterdam, The Netherlands.E-mail:n.vanmieghem@erasmusmc.nl
} 


\section{Introduction}

The surge of catheter-based valve therapies has boosted the advancement and implementation of three-dimensional (3D) imaging modalities. Multislice computed tomography (MSCT) is now pivotal for pre-procedural planning of transcatheter aortic valve implantation $(\mathrm{TAVI})^{1,2}$. The mitral valve is a more complex anatomical entity and the adoption of catheter-based mitral therapies has somewhat lagged behind the overall success of TAVI ${ }^{3}$. The edge-to-edge MitraClip (Abbott Vascular, Santa Clara, CA, USA) is arguably the single globally dispersed catheter-based technique to address mitral regurgitation. Furthermore, transcatheter closure of paravalvular leaks complicating surgically replaced mitral (and aortic) valve prostheses has become an increasingly popular strategy ${ }^{4}$. Echocardiography is the cornerstone of pre-procedural planning for these mitral valve procedures; conversely, fluoroscopy and particularly transoesophageal echocardiography (TOE) provide per-procedural guidance. TOE is invaluable for navigating wires and catheters through the interatrial septum and paravalvular leaks or for MitraClip positioning, grasping and release. Acquisition of steady two-dimensional (2D) or 3D TOE images can sometimes be challenging, time-consuming and can prolong the overall procedure time. Pre-procedural cardiac MSCT may offer unique 3D perspectives to facilitate fluoroscopy guidance of mitral valve procedures. The 3mensio Structural Heart (Pie Medical Imaging BV, Maastricht, The Netherlands) planning software has inherent features that could help localise anatomical entities by fluoroscopy which would otherwise be invisible (e.g., interatrial septum, paravalvular leaks, mitral leaflets), and importantly it could provide optimal C-arm gantry angles to facilitate crucial steps of catheter-based mitral interventions.

\section{Current needs}

\section{INTERATRIAL SEPTUM BY FLUOROSCOPY}

The interatrial septum and the mitral leaflets are radiolucent and thus fluoroscopically invisible, which partially explains why echocardiography, and TOE in particular, is the driving imaging modality for catheter-based mitral valve procedures. When using a venous transseptal approach for complex mitral interventions, identification of the optimal puncture site through the interatrial septum is crucial, as it will allow adequate manoeuvring of steerable catheters within the left atrium. In principle, a relatively superior and posterior septum crossing is required and typically the integration of three TOE planes provides guidance: the bicaval view ( $\cong 90-120^{\circ}$ ) depicts the craniocaudal orientation and facilitates superior crossing, a short-axis view $\left(\cong 30-60^{\circ}\right)$ at the base typically displaying the aortic valve provides anterior-posterior orientation to confirm sufficient posterior location, and the fourchamber view $\left(\cong 0^{\circ}\right)$ helps to determine the height above the mitral valve (Figure 1). 3D TOE may confirm the correct puncture site.

\section{MITRACLIP ORIENTATION}

A crucial step in MitraClip implantation is the accurate orientation of the clip perpendicular to the line of coaptation of the mitral valve prior to entering the left ventricle for final leaflet grasping 5 . Proper MitraClip alignment is obtained by opening the clip in the

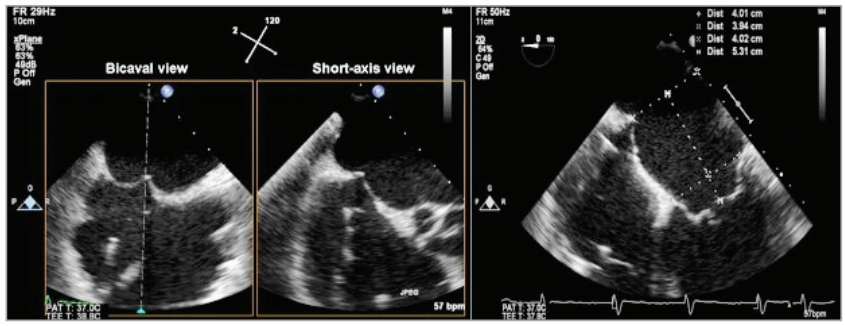

Figure 1. Transseptal puncture with tenting using three standard transoesophageal echocardiography views. Bicaval and short-axis views (left and middle panels) as acquired with 2D biplane imaging, with the secondary image "right inverted" to correspond with the short-axis view of the aortic valve at approximately 45 degrees. In the four-chamber view (right panel) the height of the tented septum to the mitral annulus is measured.

left atrium above the mitral valve. The clip delivery system should be manipulated and rotated so that the MitraClip arms are visible in full length in the long-axis view but in profile (no arms visible) in the intercommissural view (Figure 2).

\section{PERIPROSTHETIC LEAKS BY FLUOROSCOPY}

In the cathlab, the exact location of a periprosthetic leak can be challenging and requires TOE imaging ${ }^{6}$. A combination of 2D and, in particular, 3D TOE is helpful in orientating a steerable guide catheter towards the leak to help traverse the leak with a guidewire and a catheter. The acquisition of proper TOE views can be challenging for various reasons, e.g., imaging artefacts or a variable orientation of the heart in the thoracic cavity.

\section{MSCT-predicted angiographic projections}

The interatrial septum, the mitral valve apparatus or periprosthetic leaks can be identified by MSCT. It is possible to determine angiographic views coaxial and perpendicular to these structures and identify radiopaque (anatomical) landmarks that could help locate these radiolucent structures by fluoroscopy.

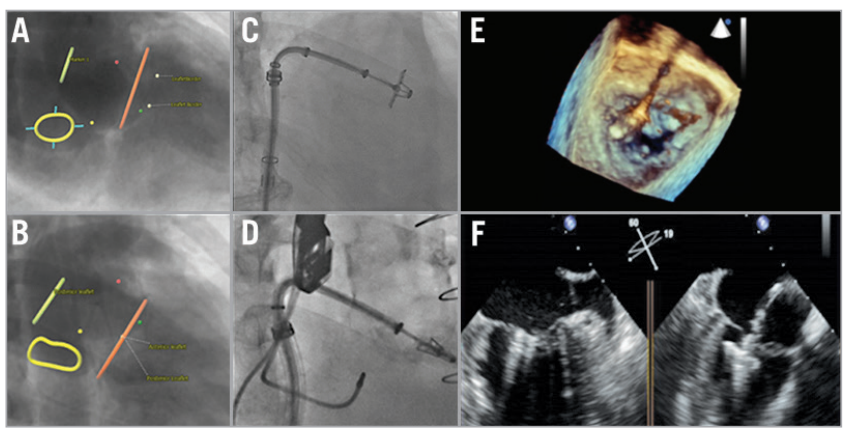

Figure 2. MitraClip orientation. Angio simulations by MSCT provide an optimal C-arm angulation coaxial (A) and perpendicular (B) to the mitral coaptation plane. C) Confirmation of the opened MitraClip en face in the suggested fluoroscopy projection from panel A. D) MitraClip in profile when using the suggested projection in panel B. Panels $E$ and F illustrate a correct orientation of the MitraClip prior to entering the left ventricle using $3 D(E)$ and $2 D$ (F) transoesophageal echocardiography. 


\section{MSCT IMAGE ACQUISITION}

We use second- or third-generation dual-source CT scanners (SOMATOM Definition Flash and SOMATOM Force; Siemens AG, Munich, Germany) with a high-pitch acquisition mode combined with ECG triggering to obtain images in diastole, collimation $2 \times 64 \times 0.6 \mathrm{~mm}$ or $2 \times 96 \times 0.6 \mathrm{~mm}$, rotation time 285 and $250 \mathrm{~ms}$ and pitch 3.2. Reference settings for tube voltage and current modulation are $120 \mathrm{KV}$ and $190 \mathrm{mAs} /$ rotation. Alternatively, in some patients we used a standard CT acquisition protocol, as used for coronary imaging, employing prospectively or retrospectively ECG-gated acquisitions that may acquire images in multiple phases of the cardiac cycle.

From the survey image the scan region is set to include the entire heart. No beta-blockers or nitroglycerine are administered prior to the scan. Non-ionic iodinated contrast material (Visipaque ${ }^{\mathrm{TM}}$; GE Healthcare, Princeton, NJ, USA) is injected in an antecubital vein $(60 \mathrm{ml}$ at $4 \mathrm{ml} / \mathrm{s}$ for second-generation scanners and $50 \mathrm{ml}$ at $4 \mathrm{ml} / \mathrm{s}$ for third-generation scanners) and followed by a saline flush of $40 \mathrm{ml}$ at $4 \mathrm{ml} / \mathrm{s}$. Image acquisition starts with a bolus triggering technique with the region of interest placed in the left atrium, followed by a second acquisition with a limited scan range to include the left atrial appendage 25 seconds later. Images are reconstructed at $1 \mathrm{~mm}$ slice thickness with $0.6 \mathrm{~mm}$ intervals.

\section{MSCT ANALYSIS}

The 3mensio Structural Heart (prototype version 7.2) is a softwareplanning tool for catheter-based structural heart interventions and contains dedicated modules with intuitive graphical user interface. The software enables semi-automatic assessment of the mitral valve through multiplanar reconstruction (MPR) and volume rendering (VR) techniques. The saddle-shaped annulus can be traced in 3D. Cross-sectional views can be used for diameter, area and perimeter measurements. Stretched multiplanar reconstructions allow fast and accurate length measurements. The simulated angio view helps determine optimal $\mathrm{C}$-arm angles that give a projection with a perpendicular view on a given anatomical structure of choice ${ }^{7}$. In fact, for any given anatomical structure a line of perpendicularity can be drawn representing an infinite combination of RAO-LAO with cranial-caudal angles. Safety and ergonomic considerations drive the angulation eventually selected to be used in the cathlab. Steep angles are typically avoided because of radiation concerns and physical impracticalities, preserving sufficient accessibility to the working field (i.e., the right groin area).

\section{MITRAL VALVE}

The saddle-shaped mitral annulus can be traced in 3D. The long axis can be rotated along a virtual line drawn from the apex through the centre of the mitral valve. Subsequently, a plane parallel to the mitral valve can be reconstructed. The anterior and posterior mitral leaflets are colour-coded, and both anterolateral and posteromedial commissures and the line of coaptation are visible. An optimal working projection can be constructed. Typically, the mitral valve is oriented in space cranially, to the left and posteriorly, which explains an anticipated (steep) RAO and cranial or caudal angulation of the $\mathrm{C}$-arm to be perpendicular or coaxial to the mitral coaptation plane, respectively.

\section{TRANSSEPTAL CROSSING SITE}

The level of the transseptal puncture for mitral valve interventions is typically 3.5 to $4 \mathrm{~cm}$ above the mitral annulus/coaptation level. In the long-axis plane, a distance 3.5 to $4 \mathrm{~cm}$ above the line of mitral coaptation into the left atrium can be identified and annotated with a virtual marker (Figure 3). Double-oblique MPR allows visualisation of the interatrial septum. A bull's eye is created on the interatrial septum in the exact same plane with the virtual marker positioned earlier 3.5 to $4 \mathrm{~cm}$ above and coaxial to the mitral annular plane. The 3 mensio software generates an optimal projection for the $\mathrm{C}$-arm gantry axial to the interatrial septum so that the bull's eye and the virtual marker overlap. The non-coronary aortic cusp (that can be identified with a pigtail catheter on fluoroscopy) and the spine serve as additional anatomical landmarks during fluoroscopy.

\section{MITRACLIP ORIENTATION GUIDANCE}

The orientation of the opened MitraClip should be perpendicular to the line of mitral coaptation for optimal grasping of the mitral leaflets. A C-arm gantry projection could be chosen that would be either coaxial or perpendicular to this line of coaptation to visualise the opened MitraClip in profile or en face with both arms at full length, respectively (Figure 2). This principle enables rotation of the MitraClip into the required orientation solely relying on fluoroscopy. Table 1 summarises how the MSCT analysis could provide workable angio simulations for transseptal crossing and MitraClip orientation. Only high-quality MSCT studies seem appropriate.

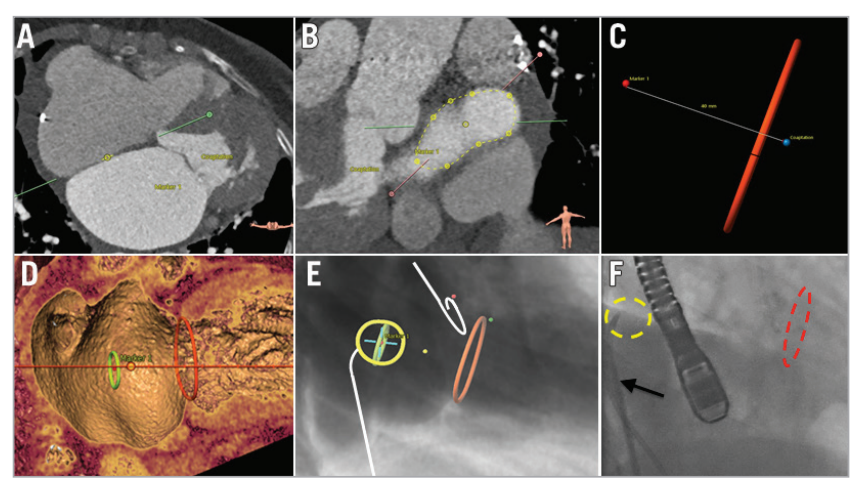

Figure 3. Transseptal crossing planning and simulation with MSCT. The fossa ovalis is identified by multislice computed tomography (MSCT) (A) and traced (B). The mitral annulus and a coaxial plane $4 \mathrm{~cm}$ into the left atrium are marked $(C \& D)$. An optimal projection can be obtained so that a target (a.k.a., bull's eye) is created that superimposes the interatrial septum and the coaxial plane $4 \mathrm{~cm}$ above the mitral annulus (E). The yellow, red and green dots identify the three aortic cusps at their widest level. In panel $F$ the real-time fluoroscopy in the proposed C-arm projection corresponds with the angio simulation by MSCT as shown in panel E. The arrow in panel $F$ marks the Mullins sheath. 
Table 1. Multislice computed tomography planning and generation of workable angio simulations for transseptal puncture and MitraClip orientation.

\begin{tabular}{|l|c|c|c|c|c|c|}
\hline & $\begin{array}{c}\text { Fluoroscopy time } \\
\text { (minutes) }\end{array}$ & MSCT quality & $\begin{array}{c}\text { Accuracy of MSCT simulation } \\
\text { for transseptal puncture }\end{array}$ & $\begin{array}{c}\text { Accuracy of MSCT simulation } \\
\text { for MitraClip orientation }\end{array}$ & $\begin{array}{c}\text { Number of } \\
\text { MitraClips }\end{array}$ & $\begin{array}{c}\text { Concomitant structural } \\
\text { heart intervention }\end{array}$ \\
\hline Patient 1 & 55.83 & Good & Good & Good & 2 & No \\
\hline Patient 2 & 27.7 & Good & Good & Good & 1 & LAA closure \\
\hline Patient 3 & 17.27 & Acceptable & Moderate & Good & 2 & No \\
\hline Patient 4 & 51 & Good & Good & Good & 2 & No \\
\hline Patient 5 & 24.42 & Good & Good & Good & 1 & No \\
\hline Patient 6 & 23.53 & Good & Good & No \\
\hline
\end{tabular}

\section{PARAVALVULAR LEAKAGE CLOSURE APPLICATION}

Double-oblique multiplanar and volume-rendered MSCT reconstructions allow the location of periprosthetic leaks. The derived angio view can help localise this leak relative to the metallic prosthesis at any given C-arm angle (Figure 4).

\section{Conclusion}

MSCT is a valuable adjunct to pre-procedural planning for catheter-based mitral valve interventions and may be complementary to TOE. Dedicated planning software offers intuitive tools to determine optimal C-arm angulations and help localise anatomical entities by fluoroscopy. Systematic use and concise analysis of MSCT may expedite these complex mitral interventions.

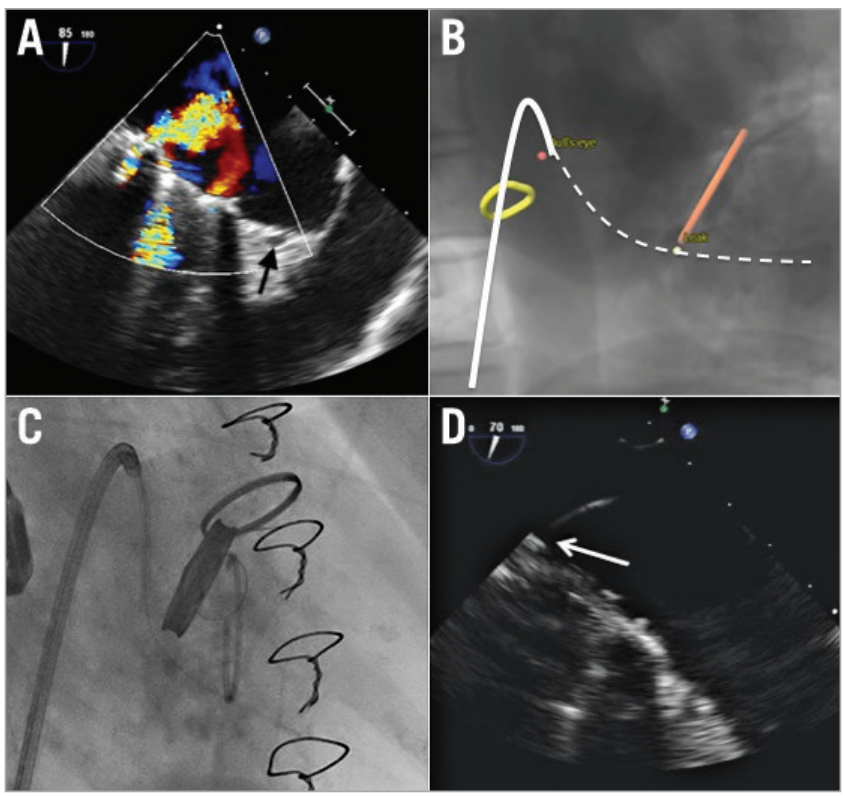

Figure 4. Periprosthetic mitral valve leakage. A) The periprosthetic leak at the posteromedial commissure by 2D TOE; the arrow indicates the coronary sinus. B) The leak (yellow dot) relative to the prosthesis in a given $C$-arm angulation; the solid white line corresponds with the steering catheter and the dashed line with the guidewire from panel $C$. C) Confirmation of the periprosthetic leak location by real-time fluoroscopy in the proposed projection. D) Through a steering catheter (arrow) a wire is navigated across the leak at the level of the posteromedial commissure. D) The arrow points towards the catheter through the periprosthetic leak (zoomed image).

\section{Impact on daily practice}

Catheter-based mitral interventions require careful pre-procedural planning. Multislice computed tomography can help determine optimal $\mathrm{C}$-arm angulations during fluoroscopy to 1) expedite and facilitate transseptal crossing, 2) identify the mitral coaptation plane, and 3) help locate periprosthetic leaks.

\section{Conflict of interest statement}

J. Vogelaar and L. Verstraeten are employees of 3mensio Medical Imaging BV. The other authors have no conflicts of interest to declare.

\section{References}

1. Schultz C, Moelker A, Tzikas A, Piazza N, de Feyter P, van Geuns RJ, Serruys PW, Krestin GP, de Jaegere P. The use of MSCT for the evaluation of the aortic root before transcutaneous aortic valve implantation: the Rotterdam approach. EuroIntervention. 2010;6:505-11.

2. Bloomfield GS, Gillam LD, Hahn RT, Kapadia S, Leipsic J, Lerakis S, Tuzcu M, Douglas PS. A practical guide to multimodality imaging of transcatheter aortic valve replacement. JACC Cardiovasc Imaging. 2012;5:441-55.

3. Van Mieghem NM, Piazza N, Anderson RH, Tzikas A, Nieman K, De Laat LE, McGhie JS, Geleijnse ML, Feldman T, Serruys PW, de Jaegere PP. Anatomy of the mitral valvular complex and its implications for transcatheter interventions for mitral regurgitation. $\mathrm{J} \mathrm{Am} \mathrm{Coll} \mathrm{Cardiol.}$ 2010;56:617-26.

4. Kliger C, Eiros R, Isasti G, Einhorn B, Jelnin V, Cohen H, Kronzon I, Perk G, Fontana GP, Ruiz CE. Review of surgical prosthetic paravalvular leaks: diagnosis and catheter-based closure. Eur Heart J. 2013;34:638-49.

5. Debonnaire P, Delgado V, Bax JJ, Marsan NA. Tools \& Techniques - Clinical: 3D transoesophageal echocardiography for selecting and guiding in percutaneous mitral valve repair using MitraClip. EuroIntervention. 2014; 10:884-6.

6. Gafoor S, Steinberg DH, Franke J, Bertog S, Vaskelyte L, Hofmann I, Sievert H. Tools and techniques--clinical: paravalvular leak closure. EuroIntervention. 2014;9:1359-63.

7. Samim M, Stella PR, Agostoni P, Kluin J, Ramjankhan F, Budde RP, Sieswerda G, Algeri E, van Belle C, Elkalioubie A, Juthier F, Belkacemi A, Bertrand ME, Doevendans PA, Van Belle E. Automated 3D analysis of pre-procedural MDCT to predict annulus plane angulation and $\mathrm{C}$-arm positioning: benefit on procedural outcome in patients referred for TAVR. JACC Cardiovasc Imaging. 2013;6:238-48. 